\title{
Ablación por radiofrecuencia de lesiones renales: experiencia inicial en un centro de referencia en la ciudad de México (INNSZ)
}

\author{
Vázquez-Lavista LG*, Flores-Balcazar $\mathrm{CH}^{* * *}$, Méndez-Probst $\mathrm{CE}^{*}$, Gabilondo-Pliego B*, \\ Sotomayor-de-Zavaleta M*, Feria-Bernal G*, Cabrera-Aleksandrova T**. \\ *Departamento de Urología. **Departamento de Radiología Intervencionista. ***Departamento de Oncología. \\ Instituto Nacional de Ciencias Médicas y Nutrición Salvador Zubirán. México.
}

Actas Urol Esp. 2008;32(10):985-988

\section{RESUMEN}

ABLACIÓN POR RADIOFRECUENCIA DE LESIONES RENALES: EXPERIENCIA INICIAL EN UN CENTRO DE REFERENCIA EN LA CIUDAD DE MÉXICO (INNSZ)

Introducción y objetivos: La incidencia de los tumores renales ha aumentado, debido con mucho, a los avances de la tecnología, pero el estadio al momento del diagnóstico ha disminuido. En pacientes seleccionados puede realizarse nefrectomía parcial (o ahorradora de nefronas). Hay pacientes que tienen co-morbilidades importantes que no son candidatos a tratamiento quirúrgico; para ellos hay modalidades nuevas de mínima invasión que utilizan diferentes tipos de energía para realizar ablación del tejido neoplásico, una de éstas es la ablación por radiofrecuencia. Este artículo reporta la experiencia inicial con esta modalidad terapéutica en un centro de referencia de la ciudad de México.

Métodos: Cuatro pacientes con 5 lesiones renales menores a $3 \mathrm{~cm}$, con comorbilidades importantes que los excluían de posibilidad quirúrgica, se sometieron a ablación por radiofrecuencia bajo guía fluoroscópica.

Resultados: cinco lesiones renales en cuatro pacientes sometidos a ablación por radiofrecuencia en un periodo de 18 meses. No se presentaron complicaciones importantes durante el procedimiento, el seguimiento promedio de 12 meses y mantienen buen control oncológico.

Conclusiones: La ablación por radiofrecuencia es una opción terapéutica segura y reproducible para pacientes con lesiones renales incidentales menores a $3 \mathrm{~cm}$. Se requiere de más tiempo para comparar el control oncológico con el estándar de oro, la cirugía.

Palabras clave: Ablación por radiofrecuencia. Cáncer renal. Lesiones renales incidentales.

\section{ABSTRACT \\ RADIOFREQUENCY ABLATION OF RENAL LESIONS: INITIAL EXPERIENCE IN A CENTER OF REFERENCE IN MEXICO CITY (INNSZ)}

Introduction and objectives: With the advances of technology, the incidence of renal cancer has increased, but the stage at the time of diagnosis has decreased. In selected patients nephron sparing surgery can be made but there are patients who have important comorbidities and therefore they are not candidates to surgical management. For them, there are new minimally invasive procedures that use different types of energy to perform ablation of the neoplastic tissue; one of which is radiofrequency ablation. This article reports the initial experience with this therapeutic modality in a reference center of Mexico City.

Methods: Four patients with 5 renal masses of $3 \mathrm{~cm}$ or less, and with insignificant comorbidities that exclude the possibility of surgery, underwent radiofrequency ablation under fluoroscopic guidance.

Results: Five renal tumors in four patients were treated with radiofrequency ablation in a period of 18 months. No major complication during the procedure was reported, mean follow- up was of 12 months and they keep a good oncologic control.

Conclusions: Radiofrequency ablation is a safe and reproducible therapeutic option for patients with incidental renal masses below $3 \mathrm{~cm}$. We need more follow up to compare oncologic control with the gold standard, surgery. Keywords: Radiofrequency ablation. Renal cancer. Incidental renal masses. 
$\mathrm{E}$ 1 cáncer de riñón es la séptima neoplasia maligna en hombres y la doceava en mujeres en los estados unidos y representa el $2,6 \%$ de todos los cánceres $^{1}$. La incidencia de los tumores renales ha aumentado, debido con mucho, a los avances de la tecnología, pero el estadio al momento del diagnóstico ha disminuido. Así, en la actualidad, lo común es encontrar masas renales pequeñas -menores de $3 \mathrm{~cm}$ - cuya característica es el crecimiento lento y ser poco proclives a la metástasis ${ }^{2-5}$. Las opciones terapéuticas para estos tumores van desde nefrectomía parcial laparoscópica hasta vigilancia, pasando por modalidades de mínima invasión que utilizan como principio ablación del tejido con diversos tipos de energía ${ }^{6}$. Como ejemplos de estas últimos se encuentra la crioterapia, la terapia térmica con microondas, el ultrasonido focal de alta intensidad y la ablación por radiofrecuencia ${ }^{7,8}$.

La radiofrecuencia como tratamiento para cáncer renal fue introducida en 1997 por Zlotta ${ }^{9}$. Su fundamento es la destrucción del tejido a base de calor, mediante el empleo de corriente eléctrica alterna que llega a alcanzar altas frecuencias (mas de $400.00 \mathrm{~Hz}$ ). La energía se aplica bajo monitorización de temperatura o de impedancia (la oposición total de corrientes alternas por un circuito eléctrico). La energía emitida por esta técnica tiene como resultado una agitación iónica en los tejidos circundantes al lugar de aplicación de la sonda de radiofrecuencia. Esto genera calor que asciende hasta los $49^{\circ} \mathrm{C}$ que es el responsable de la muerte celular por desnaturalización de proteínas, daño directo a la membrana y destrucción térmica del citoplasma ${ }^{10}$.

Ciertamente, esta modalidad terapéutica se encuentra en fase de experimentación, con series pequeñas de entre 5 y 20 pacientes, y únicamente una en que se han tratado a 100 pacientes. Las indicaciones propuestas de este tratamiento son una condición co-mórbida que contraindique ciru- gía, edad avanzada, expectativa de vida mayor a un año pero menor a 10 años, riñón solitario, enfermedad de Von Hippel Lindau. Se recomienda en lesiones pequeñas y periféricas, si bien se han reportado casos de lesiones centrales con buenos resultados, toda vez que se utilice irrigación con solución glucosada fría para evitar daño a sistemas colectores ${ }^{9}$.

El objetivo de este trabajo es reportar nuestra experiencia inicial de cinco lesiones en cuatro pacientes en un centro de referencia de tercer nivel en la ciudad de México.

\section{MÉTODOS}

Se estudiaron 4 pacientes con cáncer renal cuyas características demográficas se muestran en la Tabla 1. En los cuatro enfermos la ablación por radiofrecuencia se realizó de forma ambulatoria. El paciente se coloca en posición de decúbito dorsal o ventral y se realiza asepsia y antisepsia de la región. Se aplica anestésico local y sedación intravenosa, posteriormente se introduce la aguja de radiofrecuencia tipo LeVeen (Fig. 1) mediante guía tomográfica (Fig. 2). Se localiza el diámetro mayor del tumor y se dirige la aguja hacia el borde interno de la lesión. Una vez en este sitio se realiza el impacto, que puede ser variable dependiendo de las características y estructuras adyacentes. Sin apartarse del sitio inicial, se realiza un segundo impacto 30 segundos después pero, en esta ocasión, a la mitad de la temperatura máxima del primer impacto. En un paciente $\left(\mathrm{N}^{\circ} 4\right)$ fue necesario un tercer impacto debido a la complejidad de la lesión. Se realizó una segunda sesión a un tumor residual. En todos los casos de verificó la hemostasia y los pacientes se trasladaron a la zona de recuperación.

\section{RESULTADOS}

Ninguno de los pacientes fue candidato a tratamiento quirúrgico, por presentar condiciones comórbidas. Se realizaron 5 ablaciones por radiofre-

Tabla 1

\begin{tabular}{lcllc}
\hline & Edad & Comorbilidades asociadas & Tamaño de lesión & Tiempo de seguimiento \\
\hline Paciente 1 & 84 & DM 2, de larga evolución & $3 \times 3 \mathrm{~cm}$ & $18 \mathrm{meses}$ \\
Paciente 2 & 50 & Ca próstata avanzado & $3 \times 4 \mathrm{~cm}$ & $18 \mathrm{meses}$ \\
Paciente 3 & 78 & Ca colon estadio clínico IIb & $3 \times 3 \mathrm{~cm}$ & meses \\
Paciente 4 & 86 & Hipertensión pulmonar severa, & Quiste Bosniak III de $3 \times 4 \mathrm{~cm}$ & $6 \mathrm{meses}$ \\
\hline
\end{tabular}

Abreviaturas: $\mathrm{DM}=$ Diabetes Mellitus. $\mathrm{Ca}=$ cáncer. 


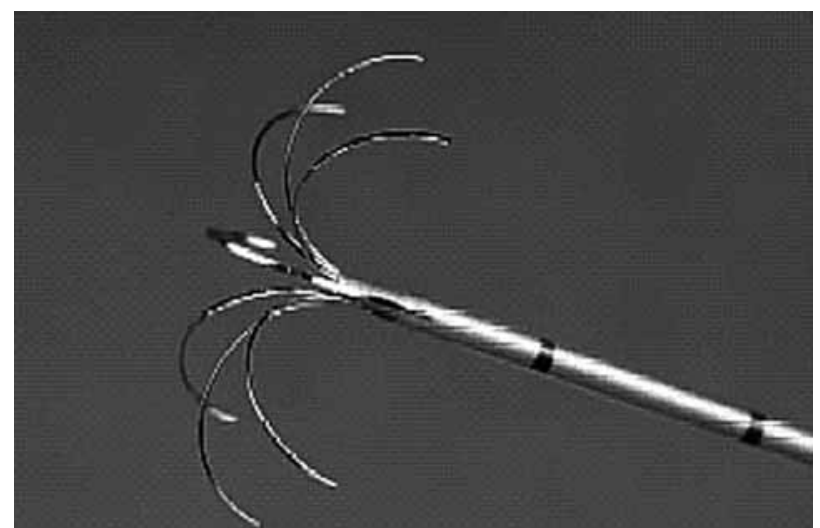

FIGURA 1. Aguja tipo LeVeen.

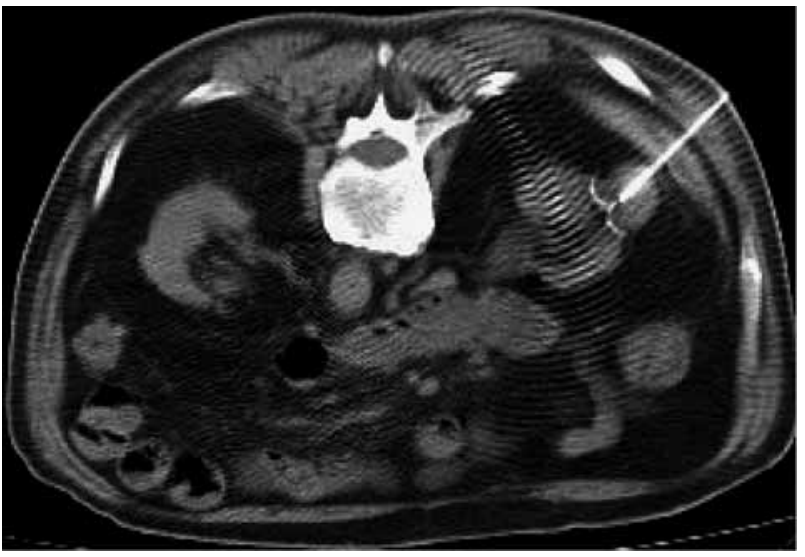

FIGURA 2. Localización vía fluroscopía de lesión renal izquierda.

cuencia (cinco lesiones renales) bajo guía fluoroscópica en 4 pacientes. El tiempo promedio de cada procedimiento fue de $45+/-15$ minutos.

En el primer paciente el tratamiento no tuvo complicaciones y a 18 meses de seguimiento no hay evidencia de actividad tumoral. En el segundo paciente fue necesario un abordaje trans-hepático ya que la lesión se encontraba por detrás del hígado, así y todo, no hubo incidentes durante el procedimiento (Fig. 3). Actualmente, 18 meses después de la intervención no hay evidencia de actividad tumoral y el cáncer de próstata se encuentra controlado con un último antígeno prostático de $0,45 \mathrm{ng} / \mathrm{ml}$. El tercer paciente lleva 10 meses de seguimiento sin evidencia de recidiva tumoral en sus dos primeras tomografías de control. En el cuarto paciente se presentó un hematoma subcapsular durante la aplicación de la radiofrecuencia el cual se manejó de forma conservadora. Después de 6 meses de seguimiento no hay evidencia de recidiva tumoral.

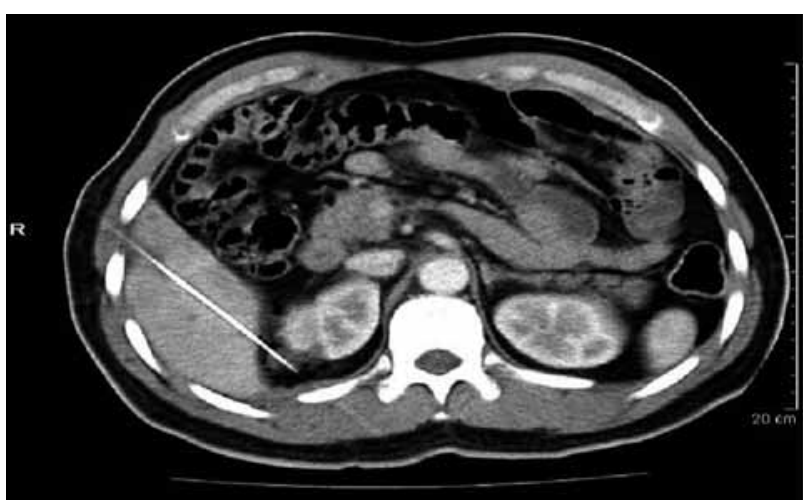

FIGURA 3. Abordaje transhepático de lesión renal derecha.

\section{DISCUSION}

La ablación por radiofrecuencia es una modalidad terapéutica que surgió por primera vez hace ya una década como una intervención experimental. Actualmente ha demostrado ser una opción terapéutica reproducible y segura. La serie más grande reportada hasta ahora fue de 100 lesiones renales, de las cuales 52 eran menores a $3 \mathrm{~cm}$ y 48 fueron mayores a $3 \mathrm{~cm}$. Las lesiones mayores de $3 \mathrm{~cm}$ requirieron más de una ablación para alcanzar la necrosis coagulativa completa ${ }^{11}$. En el análisis multivariado de esa misma serie, las dos variables que predijeron necrosis completa fueron la localización periférica y el tamaño menor a $3 \mathrm{~cm}$. El seguimiento fue de 2,3 años/paciente por lo que no puede hacerse una evaluación comparativa de manera directa con la sobrevida encontrada en pacientes sometidos a nefrectomía parcial.

Un trabajo anterior ${ }^{6}$ reportó la ablación por radiofrecuencia de 24 lesiones renales en 21 pacientes. Durante el seguimiento se advirtió que las lesiones disminuyeron de tamaño de $2,4+/-0,4 \mathrm{~cm}$ a $2,0+/-0,5 \mathrm{~cm}$, e interesantemente la mayoría de los tumores (79\%) no reforzaba después de la administración del medio de contraste. Únicamente se reportaron efectos adversos leves relacionados con el sitio de punción y dolor transitorio del psoas en dos pacientes. Los autores mencionan que la ablación por radiofrecuencia es un procedimiento seguro con pocas complicaciones.

Nuestra pequeña serie de 5 lesiones en 4 casos semeja las series mayores en cuanto a complicaciones durante el procedimiento. Si bien el seguimiento ha sido tan solo de 18 meses en dos pacientes hasta ahora el control oncológico es aceptable, sin evidencia de actividad tumoral o progresión de la 
enfermedad. Hay que recalcar que todas las lesiones que tratamos fueron menores a $3 \mathrm{~cm}$, es decir lesiones renales en estadio Tla de acuerdo a la última versión del TNM, i.e. lesiones de buen pronóstico y poco potencial para progresar y metastatizar ${ }^{12}$.

Un punto inquietante de esta nueva modalidad terapéutica es el riesgo de destruir, además de tejido tumoral, tejido sano circundante, sobre esto un trabajo reciente comparó la efectividad y el campo de necrosis coagulativa de 4 diferentes sondas de radiofrecuencia ${ }^{10}$. Los autores concluyeron que los sistemas monopolares actuales ofrecen características técnicas para controlar la ablación del tejido sin lesionar estructuras adyacentes.

Uno de los puntos más importantes es el control oncológico. El seguimiento de los pacientes después de la ablación esta basado en la realización de tomografía axial computarizada con medio de contraste intravenoso. La falta de captación del medio de contraste en el área sometida a ablación se relaciona con la necrosis coagulativa que corresponde a la muerte de las células neoplásicas. Hasta ahora, este es el único parámetro con el que se cuenta para valorar el éxito del tratamiento.

Como toda técnica innovadora la ablación por radiofrecuencia necesariamente tendrá que aprobar la prueba del tiempo. Recientemente este punto fue analizado comparando técnicas mínimamente invasivas para lesiones renales pequeñas, i.e. ablación por crioterapia y por radiofrecuencia ${ }^{13}$. Se concluye que es una técnica segura, reproducible pero que se requiere mayor tiempo de seguimiento para poderla comparar con la cirugía, que hoy por hoy, sigue siendo el estándar de oro.

\section{CONCLUSIONES}

La ablación por radiofrecuencia es una alternativa real para pacientes con alto riesgo quirúrgico, con lesiones pequeñas (menores de $3 \mathrm{~cm}$ ) y de preferencia periféricas. Es un procedimiento ambulatorio, fácilmente reproducible y con pocas complicaciones reportadas. En cuanto al control oncológico hace falta mayor tiempo de seguimiento y es necesaria la realización de estudios aleatorizados para compararla con el estándar de oro, la nefrectomía parcial.

\section{REFERENCIAS}

1. Cohen HT, Mcgovern FJ. Renal Cell Carcinoma. N Engl J med. 2005;353(23):2477-2490.

2. Nguyen MM, Gill IS, Ellison LM. The evolving Presentation of Renal Carcinoma in the United States: Trends Form the Sirveillance, Epidemiology, and End Results Program. J Urol. 2006;176(6 Pt 1):2397-2400.

3. Rendon RA, Stanietzky N, Panzarella T, Robinette M, Klotz LH, Thurston $\mathrm{W}$, et al. The natural history of small renal mases. $\mathrm{J}$ Urol. 2000;164(4):1143-1147.

4. Walther MM, Choyke PL, Glenn G, Lyne JC, Rayford W, Venzon $D$, et. al, Renal cancer in families with hereditary renal cancer: prospective analysis of a tumor size threshold for renal parenchymal sparing surgery. J Urol. 1999;161(5):1475-1479.

5. Bosniak MA. Observation of small incidentally detected renal masses Semin Urol Oncol. 1995;13(4):267-272.

6. Pavlovich P C, Walther MM, Choyke PL, Paulter SE, Chang R, Linehan W, et al. Percutaneous radio frequency ablation of small renal tumors: initial results J Urol. 2002;167(1):10-15.

7. Atwell TD, Farrell MA, Callstrom MR, Charboneau JW, Leibovich BC, Frank I, et al. Percutaneous cryoablation of large renal masses: technical feasibility and short-term outcome. AJR Am J Roentgenol. 2007; 188(5):1195-2000.

8. Janzen N, Zisman A, Pantuck AJ, Perry K, Schulam P, Belldegrun AS. Minimally invasive ablative approaches in the treatment of renal cell carcinoma. Curr Urol rep. 2002;3(1):1320.

9. Zlotta AR, Wildschutz T, Raviv G, Peny MO, van Gansbeke D, Noel JC, et al. Radiofrequency interstitial tumor ablations a possible new modalitiy for treatment of renal cancer: ex vivo and in vivo experience. J Endourol. 1997;11(4):251-258.

10. Häcker A, Vallo S, Weiss C, Grobholz R, Alken P, Knoll T, et al Minimally invasive treatment of renal cell carcinoma: comparison of 4 different monopolar radiofrequency devices. Eur urol. 2005;48(4):584-92

11. Gervais DA, McGovern FJ, Arellano RS, McDougal WS Mueller PR, Radiofrequency ablation of renal cell carcinoma: part 1 Indications, results, and role in patient management over a 6 year period and ablation of 100 tumors. AJR Am J Roentgenol. 2005;185(1):64-71.

12. Lopez-Beltran A, Scarpelli M, Montironi R, Zirkali Z. 2004 WHO classification fo the renal tumors of the adults. Eur Urol. 2006;49(5):798-805.

13. Mouravieu V, Joniau S Van Poppel H, Polascik T. Current status of minimally invasive ablative techniques in the treatment of small renal tumor. Eur Urol. 2007;51(2):328-336.

Correspondencia autor: Dr. L.G. Vázquez Lavista

Departamento de Urología

Instituto Nacional de Ciencias Médicas y Nutrición Salvador Zubirán.

Vasco de Quiroga 15. Tlalpan. 14000, México, D.F.

E-mail autor: lugavala@yahoo.com

Información artículo: Original - Cáncer renal

Trabajo recibido: marzo 2007

Trabajo aceptado: diciembre 2007 